\title{
Gênero Acosmium: composição química e potencial farmacológico
}

\author{
Paulo T. Sousa Júnior, ${ }^{*, 1}$ Evandro L. Dall'Oglio, ${ }^{1}$ Luiz Everson da Silva, ${ }^{1}$ Uir S. \\ Figueiredo, ${ }^{1}$ Paulo C. Vieira, ${ }^{2}$ Helen V. Machado, ${ }^{1}$ Luciane G. dos Santos ${ }^{1}$
}

\author{
${ }^{1}$ Departamento de Química, Instituto de Ciências Exatas e da Terra, Universidade Federal de Mato Grosso, \\ 78060-900 Cuiabá-MT, Brasil, \\ ${ }^{2}$ Departamento de Química, Instituto de Química, Universidade Federal de São Carlos, 13565-905 São Carlos- \\ SP, Brasil
}

\begin{abstract}
RESUMO: O gênero Acosmium possui 17 espécies com distribuição geográfica que se estende do sudeste do México até o Nordeste da Argentina, sendo que a maioria das espécies está localizada no Brasil. A. dasycarpum, A. panamense, A. subelegans são as espécies mais utilizadas popularmente no tratamento de enfermidades. Uma busca na literatura, resguardando aspectos químicos e farmacológicos destas plantas, indicam atividade citotóxica, ação antitérmica, efeito hipoglicêmico, bem como tem sido usada no tratamento da doença de Alzheimer e desordens no sistema nervoso central. Investigação fitoquímica resultou principalmente no isolamento de terpenos, ácido cafêico, alcalóides do tipo diaza-adamantano e quinolizidínicos bem como pironas.
\end{abstract}

Unitermos: Acosmium dasycarpum, Acosmium panamense, Acosmium subelegans, Fabaceae, medicina popular, composição química, efeito farmacológico.

\begin{abstract}
Acosmium genus: chemical composition and pharmacological potential”. The genus Acosmium is composed by c.a. 17 species, with geographic distribution from southeastern Mexico to Northwestern Argentina. Most of the species, however, are located in Brazil. A. dasycarpum, A. panamense, A. subelegans are used in folk medicine to treat several ailments. A search in the literature regarding the chemical and pharmacological aspects of these plants indicates cytotoxic activity, antithermal and hypoglycemic effects, as well as their use to treat Alzheimer's disease and CNS disorders. Phytochemical investigations resulted mainly in the isolation of terpenes, caffeic acid, diaza-adamantane and quinolizidines alkaloids as well as pyrones.
\end{abstract}

Keywords: Acosmium dasycarpum, Acosmium panamense, Acosmium subelegans, Fabaceae,

folk medicine, chemical composition, pharmacological effects.

\section{INTRODUÇÃO}

As plantas foram os primeiros recursos utilizados pelo homem tanto como alimento como fonte de recursos terapêuticos. O mais provável é que, pela experimentação direta, as populações humanas foram aprendendo a distinguir as plantas úteis e de efeito benéfico, daquelas sem qualquer efeito terapêutico ou tóxico, bem como o processamento e combinações que poderiam ser usados para a obtenção de resultados positivos (Simões et al., 2000).

Com a demanda cada vez mais crescente da utilização de plantas medicinais na cura ou prevenção de doenças, e devido ao uso popular e mesmo tradicional não serem suficientes para validar cientificamente as plantas medicinais como recurso terapêutico eficaz e seguro, tornaram-se intenso o interesse de pesquisadores em conhecer os verdadeiros potenciais químicos e biológicos responsáveis por tais ações.

Na ciência moderna, o uso da manipulação de plantas resulta em alternativa eficaz para incrementar a produção de compostos valiosos no desenvolvimento de novos medicamentos. A pesquisa sistemática para obtenção de novos compostos com finalidade terapêutica pode ser executada por meio de vários processos, sendo que os mais utilizados são: síntese de novas substâncias e a modificação molecular de substâncias naturais e/ou sintéticas com propriedades farmacológicas definidas, assim como a extração, o isolamento e a 
identificação de novos compostos de fontes naturais. Nesse sentido, as fontes de origem vegetal, apresentam um atrativo inigualável na busca por substâncias ativas que possibilitem o descobrimento de novos potenciais fármacos.

Ao se considerar a perspectiva de obtenção de novos fármacos, o Brasil se destaca como o país de maior diversidade genética vegetal do mundo (Dias, 1996), com um vasto manancial ainda por ser explorado sob o ponto de vista químico-farmacológico (BarbosaFilho et al., 2007; Saúde-Guimarães \& Faria, 2007, Corrêa et al., 2008; Quintans-Júnior et al., 2008; Sousa et al., 2008). Um dos gêneros que vem se destacando na pesquisa científica, apresentando resultados químicos e biológicos amplamente satisfatórios é o Acosmium (Fabaceae). Este gênero ocorre em vasta região latinoamericana, possuindo 17 espécies, tendo como habitat desde o sudeste do México até o nordeste da Argentina (Nuzillard et al., 1999; Bridgewater et al., 1997), sendo que a maioria das espécies encontra-se no Brasil (Lewis, 1987; Polhill, 1981).

As espécies mais utilizadas, do ponto de vista da medicina popular, são A. dasycarpum, A. panamense e A. subelegans. Com indicação popular desde a ação antitérmica até depressora do sistema nervoso central (SNC) e, em alguns casos, anticancerígena e hipoglicêmica, estas plantas, vêm sendo utilizadas sob a forma de infusão das folhas, flores, casca da raiz e do caule (Afiatpour, 1990; Balandrin, 1982; Trevisan, 2002).

Considerando o uso popular acima descrito e a predominante ocorrência, no gênero, de alcalóides biologicamente ativos sob o SNC e úteis no tratamento da doença de Alzheimer (Danieli et al., 1999), este trabalho apresenta as principais espécies de Acosmium já estudadas e descritas na literatura, bem como seus respectivos constituintes químicos (Figura 1 e 2) e propriedades biológicas.

\section{ASPECTOS QUÍMICOS E BIOLÓGICOS DAS ESPÉCIES DE ACOSMIUM}

\section{Acosmium panamense (Benth.) Yakovlev}

Acosmium panamense (Benth.) Yakovlev (Fabaceae) é uma árvore de grande porte com até 40 $\mathrm{m}$ de altura, típica de floresta tropical. É conhecida popularmente como cáscara amarga, bálsamo amarelo e quina silvestre. A casca do caule e da raiz é utilizada tradicionalmente no México e Belize no tratamento contra dores estomacais, diarréia sífilis e malária (Nuzillard et al., 1999; Afiatpour, 1990; Balandrin, 1982). Já no Brasil, as flores são usadas como antitérmico, no combate a enxaqueca, asma e desequilíbrios neurovegetativos (Costa, 2001). O efeito hipoglicêmico foi observado por via oral com o extrato aquoso nas doses de 20 e $200 \mathrm{mg} / \mathrm{kg}$ e com o extrato butanólico pela mesma via nas doses de 20 e $100 \mathrm{mg} /$ $\mathrm{kg}$, revelando uma diminuição nos níveis de glicose em ratos diabéticos num período de 180 minutos. No presente trabalho também foram isolados três pironas 6[(E)-2-(4-hidroxifenil)vinil]-4-metoxi-2H-piron-2-ona (1), $\beta$-D- $O$-glicosideo (2), $\beta$-D- $O$-di(1-6)glicosídeo (3) e o ácido cafeíco (4) (Andrade-Cetto \& Wiedenfeld, 2004).

$\mathrm{Na}$ investigação química das cascas das raízes de $A$. panamense foram isolados três alcalóides quinolizidínicos metoxilados $4 \alpha$-hidróxi-13 $\beta$ metóxilupanina (5), $3 \beta, 4 \alpha$-diidróxi-13 $\beta$-metóxilupanina (6) e o 13ß-metóxilupanina (7), já descritos na literatura (Veitch et al., 1997). Neste mesmo estudo, através de ensaio biomonitorado do extrato etéreo das sementes, foram isolados sete compostos, dos quais os alcalóides lupanina (8) e multiflorina (9) foram identificados, comparando-se os dados espectrais com compostos previamente descritos na literatura (Veitch et al.,

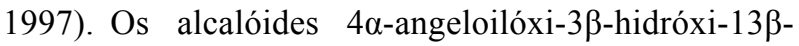
metóxilupanina (10), angustifolina (11), acosmina (12), acetato de acosmina (13) e panacosmina (14) foram descritos pela primeira vez, apresentando os três últimos um esqueleto diaza-adamantano (Veitch et al., 1997).

Em estudos realizados com o extrato metanólico da casca do caule, isolou-se pela primeira vez o composto $4 \alpha$-hidroxiesparteína (15), sendo a estrutura e a estereoquímica elucidada por comparação espectral com amostras autênticas de $4 \alpha$-acetoxiesparteína, $4 \beta$-acetoxiesparteína por CG-EM (Balandrin, 1982).

Do caule foram isolados a esparteína (16), afilina (17), homo-sweetinina ou (+/-)homo-6-epipodopetalina (18), acosminina (19), 4-Metóxi-6-( $p$-hidroxiestiril)$\alpha$-pirona (1) e lupeol (20). Das raízes, caule e folhas, isolou-se a sweetinina ou (+/-)6-epipodopetalina (21). E das raízes foi isolado a homopodopetalina (22) (Nuzillard et al., 1999; Balandrin, 1982; Veitch et al., 1997).

\section{Acosmium dasycarpum ( Vog.) Yakovlev}

Acosmium dasycapum é uma planta característica e exclusiva dos cerrados e cerradões, restrita as regiões central e nordeste do Brasil, tendo ocorrência no cerrado brasileiro entre os estados da Bahia, Minas Gerais, São Paulo, Mato Grosso e Goiás. Sua altura varia entre 4 e $6 \mathrm{~m}$, dotada de copa pequena, tronco tortuoso com casca suberosa, folhas alternadas, flores brancas e frutos legume (vagem achatada). Floresce durante os meses de novembro e dezembro. Os frutos amadurecem a partir de fevereiro (Lorenzi, 1998).

É conhecida popularmente como perobinha do campo, chapada, pau-paratudo, unha d'anta e genciana (Lewis, 1987; Lorenzi, 1998; Rodrigues, 1996), sendo atribuídas às cascas da raiz propriedades terapêuticas como tranqüilizante, hipotensor, antineoplásico, antisifílico, antireumático e no tratamento de afecções 
cutâneas, além da ação diurética atribuída às suas folhas (Trevisan, 2002; Rodrigues, 1996; Silva et al., 2001).

Isolou-se, como componente principal do extrato benzênico do tronco, o composto lupeol (20) (Rocha et al., 1980). Ensaios farmacológicos conduzidos com este extrato indicaram atividade depressora do sistema nervoso central, podendo ser a mesma explicada pela presença do lupeol. Esta substância foi testada em camundongos mostrando ação depressora do SNC, sem atividade hipnótica até a dose de $1 \mathrm{~g} / \mathrm{kg}$ (Rocha et al., 1980). Este efeito pode estar relacionado à sua característica lipossolúvel, sendo capaz de atravessar facilmente barreiras e membranas celulares (Trevisan, 2002). Além disso, a literatura relata o uso de (20) como antiinflamatório (antireumático e antigotoso), antilitíaco (Malini et al., 2000; Geetha et al., 1999), antineoplásico (Moriarity et al., 1998; Miles \& Kopol, 1976; Sheth et al., 1973) e antimalárico (Alves et al., 1997).

Em estudos realizados por nosso grupo (Trevisan, 2002) com a casca da raiz de A. dasycarpum, foram descritos $\mathrm{o}$ isolamento $\mathrm{e}$ a identificação de alcalóides diaza-adamantanos (14) e (23) e quinolizidínicos (9), (19), (24), (25), e (26), lupeol (20) e a 4-metoxi-6-(p-hidroxiestiril)- $\alpha$-pirona (1). As substâncias (14), (19), (23), (24), (25) e (26) foram relatadas em A. dasycarpum pela primeira vez, sendo que as substâncias (23) e (24) até então eram inéditas na literatura (Trevisan, 2002). Para o extrato bruto metanólico $(\mathrm{EBMeOH})$ das cascas das raízes de A. dasycarpum, foram comprovadas as atividades antibacteriana contra a cepa Staphylococcus epidermidis e fúngica Tricophyton mentagnophytes na concentração de $50 \mu \mathrm{g} / \mathrm{mL}$ e $200 \mu \mathrm{g} / \mathrm{mL}$, respectivamente. Foi avaliado também o efeito antiinflamatório através do edema de pata induzido por carragenina, apresentando atividade após $4 \mathrm{~h}$ com $20 \mathrm{mg} / \mathrm{kg}(34 \%, \mathrm{p}<0,05)$ e $200 \mathrm{mg} / \mathrm{Kg}$ ( $58 \%, \mathrm{p}<0,001)$. Na avaliação de inibição de radicais livres, houve inibição do radical DPPH (2,2-difenil-1picrilhidrazila) quando se efetuou o experimento nas concentrações $50 \mu \mathrm{g} / \mathrm{mL}$, com uma porcentagem de inibição de $90,76 \%$. Por sua vez, no ensaio de inibição do ânion superóxido, a fração etérea foi mais ativa (50 $\mu \mathrm{g} / \mathrm{mL} ; 74 \%$ ), quando comparado com o extrato bruto $(50 \mu \mathrm{g} / \mathrm{mL} ; 47 \%)$. Através destes estudos pré-clínicos (Trevisan, 2002), foram obtidas fortes evidências no sentido da validação científica do uso popular da espécie como antiinflamatório.

$\mathrm{O} \mathrm{EBMeOH}$ bem como a fração etérea e alcaloídica, além dos compostos isolados (1) e (20), foram submetidos a testes para avaliação da citotoxicidade frente à Artemia salina Leach, apresentando resultado negativo nas concentrações 50 a $1000 \mu \mathrm{g} / \mathrm{mL}$. O resultado é considerado significativo quando a $\mathrm{DL}_{50} \leq 500 \mu \mathrm{g} / \mathrm{mL}$ (Trevisan, 2002).

Estudos realizados em nossos laboratórios com o extrato bruto metanólico do cerne das raízes, levaram ao isolamento e a identificação do triterpeno (20), da pirona (1) bem como da isoflavona (29). A atividade inibidora de radicais livres (método DPPH) também foi observada num porcentual superior a $80 \%$ (para doses de 100 e $50 \mu \mathrm{g} / \mathrm{mL}$ ). Um estudo comparativo foi executado na inibição do ânion superóxido pelo método do NBT, com resultados acima de $88 \%$ (para dose de $20 \mu \mathrm{g} / \mathrm{mL}$ ). Além disso, o composto (1) apresentou atividade antimicrobiana contra cepas de Staphylococcus epidermidis e antifúngica contra cepas de Tricophyton mentagnophytes (Parizotto, 2003).

Em trabalho anterior (Balandrin, 1982), descreveu-se o isolamento de (1) (Afiatpour, 1990; Balandrin, 1982) a partir das cascas do caule, assim como o isolamento e a identificação do lupeol (20). Alguns alcalóides quinolizidínicos do tipo esparteína, como (27) e (28), também foram isolados. Porém, a análise estrutural não foi conclusiva, e a partir das lacunas deixadas por esse autor e comparando com os dados espectroscópicos obtidos por técnicas mais modernas, comprovou-se, que tais estruturas tratavam-se na realidade dos alcalóides (24) e (26) (Trevisan, 2002). Estes alcalóides foram ensaiados em camundongos para a avaliação da atividade sobre a musculatura esquelética, cardíaca e diafragmática, observando-se potenciação da contração muscular por ação local, provavelmente explicada por uma ação intracelular, interferindo no influxo de cálcio celular (Afiatpour, 1990).

$\mathrm{O}$ extrato bruto hexânico (EBHex) e metanólico $(\mathrm{EBMeOH})$ das folhas e da casca do caule foram submetidos a triagem de atividade tripanocida e leishmanicida, sendo que nessa triagem apenas o extrato bruto hexânico da casca do caule apresentou resultado satisfatório, com $\mathrm{CI}_{50}=46,16 \mu \mathrm{g} / \mathrm{mL}$, contra formas promastigotas de L. chagasi (Machado et al., 2007).

\section{Acosmium subelegans (Mohlenbr) Yakovlev}

Acosmium subelegans é conhecida popularmente como perobinha do campo, amendoimfalso, chapadinha, sucupira-branca. A casca das raízes, do caule e folhas são utilizadas na medicina popular principalmente como antiespasmódica, antireumático, antisifílico, calmante, sedativo febrífuga na epilepsia, nos acessos da asma e no tratamento da coqueluche (Afiatpour, 1990; Balandrin, 1982; Vieira et al., 2000; Cardoso et al., 1998; De La Cruz, 1997).

$\mathrm{Na}$ avaliação toxicológica aguda e na triagem do extrato bruto etanólico da casca da raiz, quando administrado por via oral foi isento de toxicidade, e pela via intraperitoneal mostrou-se ligeiramente tóxico, com marcado efeito residual. O screening hipocrático exibiu efeitos depressores sobre o SNC, cujas manifestações principais foram: diminuição da atividade do SNC e do sistema motor bem como do tônus muscular. $\mathrm{O}$ extrato etanólico desidratado interferiu diminuindo o ganho de peso corporal e diminuição no consumo de ração quando administrado por via intraperitoneal (Araujo et 
al., 1996).

A avaliação por via oral e intraperitoneal do extrato etanólico e hidroalcoólico das cascas do caule após a administração aguda, mostrou uma atividade depressora do SNC dependente, ou seja, é função do tipo e processo de obtenção do extrato. Além disso, o efeito induzido pelo extrato etanólico é acompanhado de uma atividade anti-convulsivante (Vieira et al., 2000; Cardoso et al., 1998; Barbosa-Filho et al., 2008).

Estudos realizados com o extrato aquoso e com a fração alcaloídica potencializaram a contração dos músculos do diafragma, estimulado diretamente na presença de $d$-tubocurarina, sendo que as frações semipurificadas também mantiveram a atividade potenciadora das contrações no músculo esquelético (Cardoso et al., 1998). Estes resultados descartaram a participação dos receptores no efeito inotrópico positivo da fração e indicaram uma possível ação intracelular, além de sugerir uma provável interferência do extrato aquoso e das frações na homeostasia intracelular de cálcio (Cardoso-Lopes, 2003).

A atividade antitumoral foi observada em extratos provenientes das folhas (Antônio et al., 2000). A inibição do crescimento das células tumorais se deu na linhagem mais sensível dentre todas as analisadas. Através de estudos fitoquímicos foi observado que as folhas possuem um teor dez vezes menor de alcalóides do que as raízes (Oliveira et al., 1994). Assim, pode-se justificar a baixa sensibilidade frente às células tumorais ensaiadas.

Da casca do caule foram isolados a esparteína (16), lupeol (20), 4 $\alpha$-hidroxiesparteína (15), 5,6-deidroesparteína (30), afilina (17), angustifolina (11), tetraidrorombifolina (31), lupanina (8), sweetinina ou (+/-)6-epipodopetalina (21) e homo-sweetinina ou (+/-) homo-6-epipodopetalina (18). E das cascas do caule e raízes isolou-se as substâncias aloperina (32), multiflorina (9), $4 \alpha$-hidroxi-13-epimetoxilupanina ou $4 \alpha$-hidroxi13ß-metoxilupanina (5), (+/-)-18-epipiptantina (33), homo-18-epipiptantina (34), podopetalina (35), ormosanina (36), acosminina (19) e acosmina (12) (Nuzillard et al, 1999; Afiatpour, 1990; Balandrin, 1982; Oliveira et al., 1994).

A $\alpha$-pirona (1) foi isolada da raíz de $A$. subelegans, tendo sido também preparada pela condensação do 4-benziloxibenzaldeído com 4-metóxi6-metil- $\alpha$-pirona, em uma solução metanólica de $\mathrm{Mg}(\mathrm{OMe})_{2}$, seguida de desbenzilação com $\mathrm{HBr}$ em ácido acético (Paulino-Filho et al., 1977).

\section{Acosmium bijugum ( Yog.) Yakovlev}

Acosmium bijugum é conhecida popularmente como sucupira mirim e angico do litoral. Para a A. bijugum nenhuma indicação de uso popular é referenciada na literatura, no entanto, ensaios de atividade antiparasitária evidenciaram a atividade antimalárica para esta espécie
(Silva et al., 2001). A abordagem fitoquímica confirmou a predominância de alcalóides quinolizidínicos do tipo esparteína e ormosia (Nuzillard et al., 1999; Veitch et al., 1997; Paulino-Filho et al., 1977; Fitzgerald et al., 1964; Balandrin et al., 1981; Baisalbaeva et al., 1987).

Do extrato etanólico das cascas do caule foram isolados pterocarpanos $(\mathbf{3 7}, \mathbf{3 8}, \mathbf{3 9})$, lupeol (20), ácido betulínico (40), éster etílico do ácido caféico (41), 4-metoxi-6-( $p$-hidroxifenil)-trans-estirilpirona (42) e $\beta$-D-glicopiranosil- $\beta$-sitosterol (43) (Silva et al., 2001).

\section{CONSIDERAÇÕES FINAIS}

$\mathrm{O}$ gênero Acosmium tem mostrado um grande potencial quanto suas propriedades químicas e farmacológicas, possuindo um número expressivo de relatos no tratamento da hipertensão, afecções cutâneas, processos inflamatórios e malária além de efeito hipoglicemiante. Os estudos químico-farmacológicos relatados na literatura e os resultados obtidos em nossos laboratórios demonstram a constituição química com destaque para o isolamento do lupeol (20) em grandes quantidades, que apresenta atividade antioxidante, antiparasitária bem como o ácido betulínico (40) que é o mais efetivo agente antitumoral entre os mais de 50 derivados derivados lupanos conhecidos na literatura (Tolstikova et al., 2006). O gênero Acosmium mostrou-se uma fonte valiosa de alcalóides do tipo quinolizidínicos (Michael, 2005), além da classe das pironas (McGlacken \& Fairlamb, 2005) que são interessantes agentes antimicrobianos e do pouco usual esqueleto aza-adamantano (Barbosa-Filho et al., 2004). Este último presente na família Fabaceae possivelmente como um marcador taxonômico, de comprovada ação farmacológica como bactericida e antifúngico (Baisalbaeva et al., 1987).

Os estudos farmacológicos realizados no gênero baseiam-se, principalmente, nos extratos brutos metanólico, hexânico, etanólico, benzênico, hidroalcoólico e frações alcaloídicas. Dentre as atividades observadas predomina depressão do sistema nervoso central (Araujo et al., 1996), acompanhada de atividade antioxidante (Trevisan, 2002) e inibição do crescimento de células tumorais (Cardoso-Lopes, 2003), além de inibição e potencialização da contração muscular por bloqueio do influxo de cálcio (Afiatpour, 1990), bem como a comprovação da atividade antiparasitária (Balandrin, 1982; Sheth et al., 1973; Machado et al., 2007).

Estudos de validação dos dados etnofarmacológicos confirmam as indicações populares para as espécies de Acosmium, incluindo a atividade anti-inflamatória (Trevisan, 2002) e hipoglicemiante (Andrade-Cetto \& Wiedenfeld, 2004), antiparasitária além do combate a doenças neurodegenerativas. A atividade anticolinestérica, que implica direta relação com a doença de Alzheimer, foi investigada com o 
<smiles>[R]c1ccc(/C=C/c2cc(OC)cc(=O)o2)cc1</smiles>

1. $\mathrm{R}=\mathrm{OH}$

$\mathrm{R}=$

3. $\mathrm{R}=$

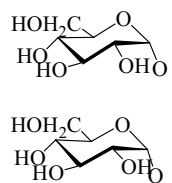

$\stackrel{\mathrm{HO}_{\mathrm{OHO}}}{\stackrel{\mathrm{C}}{\mathrm{C}}-\mathrm{O}}$<smiles>O=C(O)/C=C\c1ccc(O)c(O)c1</smiles>

4

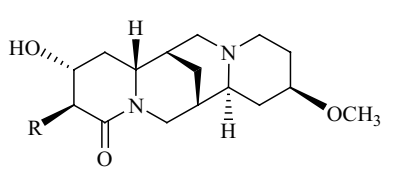

5. $\mathrm{R}=\mathrm{H}$<smiles>[R]C1CCN2C[C@@H]3CC[C@@H](CN4C(=O)CCC[C@@H]34)[C@H]2C1</smiles>

7. $\mathrm{R}=$

$\mathrm{OCH}_{3}$

8. $\mathrm{R}=\mathrm{H}$

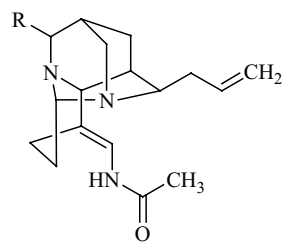

12. $\mathrm{R}$

$\mathrm{C}_{3} \mathrm{H}_{6}-\mathrm{OH}$

13. $\mathrm{R}=\mathrm{C}_{3} \mathrm{H}_{6}-\mathrm{OAc}$

14. $R$

$\mathrm{H}_{2} \mathrm{C}=\mathrm{CH}_{2}$<smiles>O=C1C=CN2CC3CC(CN4CCCCC34)C2C1</smiles><smiles>C/C=C(/C)C(=O)OC1CC2CC3CN4CCC(OC)CC4C4CC2OC1C(=O)N34</smiles>

10<smiles>C=CCC1NCC2CC1CN1C(=O)CCCC21</smiles>

11

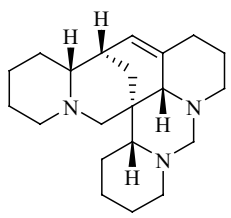

19

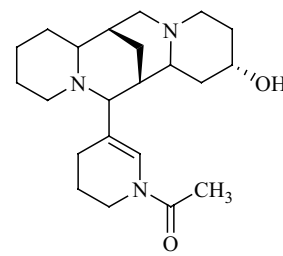

20<smiles>C1=C2/CCCCN3CN4CCCC[C@]4(C2)CN2CCCC[C@@H]2[C@H]/13</smiles>

24<smiles>COc1cc(/C=C/c2ccc(O)cc2)oc(=O)c1</smiles>

21<smiles>[Z]C1CCN2CC3CC(CN4CCCCC34)C2C1</smiles>

16. $\mathrm{R}=\mathrm{OH}$ 17. $\mathrm{R}=\mathrm{H}$ 15<smiles>O=C1CCCC2C3CC(CN12)C1CCCN3C1</smiles>

18<smiles>C1=C2CCCN[C@@H]2CCN2CCCN[C@H]12</smiles>

23<smiles>C=CCC1CCCCC2CC3C1CC2CN(C)C3C1=CN(C(C)=O)CCC1</smiles>

25

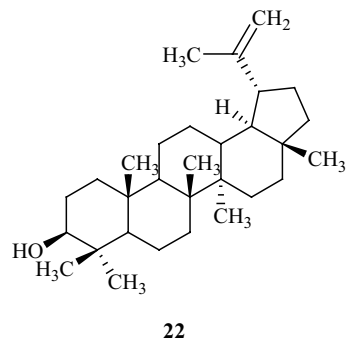<smiles>C1=C2CCCN3CN4CCCCC4C4(CCCCC24)CN2CCCCC32C1</smiles>

26

Figura 1. Constituintes químicos de Acosmium. 
<smiles>CN1C(=O)CCC2=CC3CC4(CN5CCCCC54)C3C2C1C1CCCCN1</smiles>

27<smiles>C=CCC(C(C)C)C12CN3CCCCC3C1C=C1CCC(=O)N(C)C12</smiles>

28

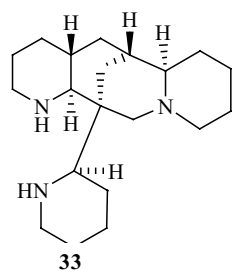<smiles>COc1cc(/C=C/c2ccc(O)cc2)oc(=O)c1</smiles><smiles>COc1cc(-c2coc3cc(O)ccc3c2=O)ccc1O</smiles><smiles>C1=C2C3CC(CN2CCC1)C1CCCCN1C3</smiles>

30<smiles>C=CCCN1CC2CC3CCCC(=O)N(CC3C2)C1</smiles>

31<smiles>C1=C2CCCNC2C[C@H]2CN3CCCCC3C12</smiles>

32<smiles>CCOC(=O)/C=C/c1ccc(O)c(O)c1</smiles>

42<smiles>COc1ccc(/C=C/c2cc(OC)cc(=O)o2)cc1</smiles>

43<smiles></smiles>

34<smiles>C1CCC(C23CN(CCCN4CCCCC42)CC24NCCCC2CC43)NC1</smiles>

35<smiles>[R9]c1ccc2c(c1Br)OCC1c3cc4c(cc3OC21)OCO4</smiles>

38. $\mathrm{R}=\mathrm{R}^{1}=\mathrm{H}$

39. $\mathrm{R}=\mathrm{H} ; \mathrm{R}^{1}=\mathrm{OMe}$

40. $\mathrm{R}=\mathrm{OMe} ; \mathrm{R}^{1}=\mathrm{OH}$

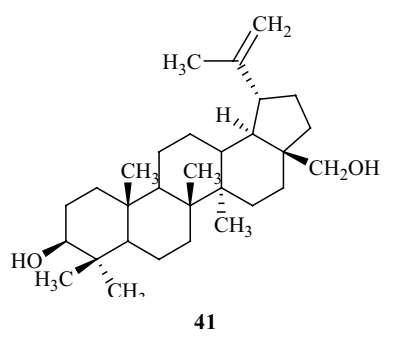

41<smiles>CC(C)C(C)CCC(C)C1CCC2C3C=CC4CC(OC5OCC(O)C(O)C(O)C5O)CCC4(C)C3CCC12C</smiles>

44

Figura 2. Constituintes químicos de Acosmium. 
alcalóide quinolizidínico (16), apresentando resultados promissores como protótipo para o tratamento do mal de Alzheimer, bem como a outras doenças relacionadas ao sistema nervoso central (Danieli et al., 1999). Pode-se ressaltar também a ação hipoglicemiante, anti-arrítmica e hipotensiva atribuída a lupanina (8), depressora do SNC conferida a multiflorina (9) e diurética, antitumoral e estimulante do sistema respiratório demonstrada pela esparteína (17) (Balandrin, 1982). Tais constatações motivam as investigações químico-farmacológicas, sobretudo na busca de novos agentes fitoterápicos seletivos a determinados alvos biológicos.

Enfim, os estudos realizados em Acosmium demonstram o valor do conhecimento tradicional para o desenvolvimento e autentificação de novos agentes medicinais. Deve-se ressaltar que o isolamento e caracterização das entidades química presentes no gênero, correlacionada com a atividade farmacológica, podem contribuir para efetiva descoberta de compostos ainda mais relevantes para a terapêutica e assim beneficiar efetivamente a sociedade.

\section{REFERÊNCIAS}

Afiatpour P 1990. Alcalóides quinolizidínicos isolados de Acosmium dasycarpum (Vog) Yakovlev: estruturas químicas e atividades farmacológicas. São Paulo, 125p. Tese de Doutorado - Escola Paulista de Medicina, Universidade Federal de São Paulo.

Alves TM, Nagm TJ, De Carvalho LH, Krettli AU, Zani CL 1997. Antiplasmodial triterpene from Vernonia brasiliana. Planta Med 63: 554-555.

Andrade-Cetto A, Wiedenfeld H 2004. Hypoglycemic effect of Acosmium panamense bark on streptozotocim diabetic rats. J Ethnopharmacol 90: 217-220.

Antônio MA, Kohn LK, Peres O, Araújo CEP, Finkelfarb E, Carvalho LE 2000. Atividade antiproliferativa dos extratos brutos de plantas da região de Bragança Paulista. $7^{\circ}$ Interamerican Congresso of Clinical Pharmacology and Therapeutics. Águas de Lindóia, Brasil.

Araujo LCL, Araujo CEP, Finkelfarb E, Nogueira DCF, Oliveira FDE 1996. Avaliação toxicológica aguda e screening hipocrático do extrato de Acosmium subelegans (Mohlemb) Yakovelev em camundongos. Lecta 14: 111-135.

Baisalbaeva AS, Omarov TT, Nikitina ET, Kazakova GG 1987. Synthesis and antimicrobial activity of diazaadamantane derivatives. KhimikoFarmatseuticheskii Zhurna 21: 191-195.

Balandrin MF, Kinghorn AD 1981. Characterization of sweetinine, a constituent of Sweetia elegans, as the Ormosia alkaloid, (*)-6-Epipodopetaline. J Nat Prod 44: 619-621.

Balandrin MF 1982. Struture elucidation of some biologically active constituents of the genus Acosmium (Leguminosae). Chigago, 117p. Tese de Doutorado - College of Pharmacy, Univercity of Illinois at the Medical Center.

Barbosa-Filho JM, Almeida JRGS, Cunha EVL, Silva MS,
Braz-Filho R 2004. Bowdichine, a new diazaadamantane alkaloid from Bowdichia virgilioides. $J$ Asian Nat Prod Res 6: 11-17.

Barbosa-Filho JM, Nascimento-Júnior FA, Tomaz ACA, Athayde-Filho PF, Silva MS, Cunha EVL, Souza MFV, Batista LM, Diniz MFFM 2007. Natural products with antileprotic activity. Rev Bras Farmacogn 17: 141-148.

Barbosa-Filho JM, Alencar AA, Nunes XP, Tomaz ACA, Sena-Filho JG, Athayde-Filho PF, Silva MS, Souza MFV, da-Cunha EVL 2008. Sources of alpha-, beta, gamma-, delta- and epsilon-carotenes: A twentieth century review. Rev Bras Farmacogn 18: 135-154.

Bridgewater SGM, Stirton, CH 1997. A morphological and biogeografhic study of the Acosmium dasycarpum complex (Leguminosae: Papilionoideae, Sophoreae). Kew Bulletin 52: 471-475.

Cardoso EM, Tanae MM, Viel TA, Souccar C, Lapa AJ, LimaLandman MTR 1998. Estudo do mecanismo de ação de frações semi-purificadas de Acosmium subelegans (Mohlenbr) Yakovlev (Perobinha do campo). $X V$ Simpósio de Plantas Medicinais do Brasil. Águas de Lindóia, Brasil.

Cardoso-Lopes EM 2003. Ação de alcalóides da Acosmium subelegans (Mohlenbr) Yakovlev no influxo e na mobilização de ions de $\mathrm{Ca}^{2+}$ em culturas primárias de músculos esqueléticos e cardíaco. São Paulo, 127p. Tese de Doutorado - Escola Paulista de Medicina, Universidade Federal de São Paulo.

Corrêa MFP, Melo GO, Costa SS 2008. Substâncias de origem vegetal potencialmente úteis na terapia da Asma. Rev Bras Farmacogn 18 (Supl.): 785-797.

Costa JBN, Rodrigues JM, Silva TM 2001. Análises da toxicidade de novos bisfosfonatos simétricos. XXIV Reunião Anual da Sociedade Brasileira de Química. Poços de Caldas, Brasil.

Danieli B, Lesma G, Passarella D, Silvani A, Viviani N 1999. Na efficient chemoenzymatic access chiral 3,7-diazabicyclo [3.3.1] nonane derivatives. Tetrahedron Lett 55: 11871-11878.

De La Cruz MGF 1997. Plantas medicinais utilizadas por raizeiros: uma abordagem etnobotânica no contexto da saúde e doença. Cuiabá, 152p. Dissertação de Mestrado - Programa de Pós-Graduação em Ciências Biológicas, Universidade Federal de Mato Grosso.

Dias BFSA 1996. Implementação da Convenção Sobre Diversidade Biológica no Brasil: desafios e oportunidades. Campinas: André Tosello.

Fitzgerald TJ, Lapidus JB, Beal JL 1964. Sweetinine, an alkaloid from Sweetia panamensis. Lloydia 27: 107110.

Geetha T, Varalashmi P 1999. Effet of lupeol and lupeol linoleate on lysosomal enzymes and collagem in adjuvant-induced arthritis in rats. Mol Cel Biochem 201: 83-87.

Lewis GP 1987. Legumes of Bahia. England: Kew Publishing. Lorenzi H 1998. Arvores Brasileiras: Manual de Identificação e Cultivo de Plantas Arbóreas do Brasil. São Paulo: Nova Odessa:Plantarum.

Machado HV, Santos LG, Silva LE, Dall'Oglio EL, Júnior PTS, Ribeiro TAN, Figueiredo US, Vieira PC, Steindel M, Pacheco LK, Nunes RK 2007. Avaliação da Atividade Antiparasitária e citotóxica da Acosmium 
dasycarpum. XXX Reunião Anual da Sociedade Brasileira de Química. Águas de Lindóia, Brasil.

Malini MM, Lenin M, Varalakshmi P 2000. Protective effects of triterpenes on calcium oxalate crystal-induced peroxidative changes in experimental urolithiasis. Pharmacol 41: 413-418.

McGlacken GP, Fairlamb IJ 2005. 2-Pyrone natural products and mimetics: isolation, characterisation and biological activity. Nat Prod Rep 22: 369-385.

Michael JP 2005. Indolizidine and quinolizidine alkaloids $N a t$ Prod Rep 22: 603-626.

Miles DH, Kokpol U 1976. Tumor inhibitors II: constituents and antitumor activity of Sarracenia flava. J Pharm Sci 65: 284-285

Moriarity DM, Huang J, Yancey CA, Zhang P, Setzer WN, Lawton RO, Bates RB, Caldera S 1998. Lupeol is the cytotoxic principle in the leaf extract of Dendropanax cf. querceti. Planta Med 64: 370-372.

Nuzillard JM, Connolly JD, Delaude C, Richard B, ZéchesAnrot M, Men-Oliver L 1999. Computer-assisted structural elucidation. Alkaloids with a novel diazoadamantane skeleton from the seeds of Acosmium panamense (Fabaceae). Tetrahedron Lett 55: 1151111518.

Oliveira F, Saito ML, Furukawa CM 1994. Caracterização farmacognóstica da droga e do extrato fluido de perobinha do campo - Acosmium subelegans. XIII Simpósio de Plantas Medicinais do Brasil. Fortaleza, Brasil.

Parizotto CA 2003. Contribuição ao estudo químico do cerne das raizes de Acosmium dasycarpum (Vog) Yakovlev. Cuiabá, 86p. Dissertação de Mestrado - Programa de Pós-Graduação em Saúde Coletiva, Universidade Federal de Mato Grosso.

Paulino-Filho HF, Muradian J, Mors WB 1997. Phytochemical study of Acosmium subelegans (Mohlenbr) Yakovlev. Isolation and synthesis of 4-methoxy-6(p-hydroxystyryl)- $\alpha$-pyrone. Rev Latinoamer Quim 8: 79-88.

Polhill RM 1981. Sophoreae. In: Advances in legume systematics. Part, Kew Publishing:England 1: 213230.

Quintans-Júnior LJ, Almeida JRGS, Lima JT, Nunes XP, Siqueira JS, Oliveira LEG, Almeida RN, AthaydeFilho PF, Barbosa-Filho JM 2008. Plants with anticonvulsant properties - a review. Rev Bras Farmacogn 18 (Supl.): 798-819.

Rocha RF, Lapa AJ, Vale JR, Braz-Filho R, Silva SB 1980. Pharmacological activity of crude and purifiede extracts from Acosmium dasycarpum (Vog) Yakvol. Cienc Cult 33: 158-162.

Rodrigues AAG 1996. Ecologia da reprodução de duas espécies de Acosmium (Schott) Benth. (LeguminosaePapilionoideae) no cerrado de Moji Guaçu, Rio Claro. 167p. Tese de Doutorado - Instituto de Biociências, Universidade Estadual Paulista.

Saúde-Guimarães DA, Faria AR 2007. Substâncias da natureza com atividade anti-Trypanosoma cruzi. Rev Bras Farmacogn 17: 455-465.

Sheth K, Bianchi E, Wiedhope R, Cole JR 1973. Antitumor agents from Alnus oregona (Betulaceae). J Pharm Sci 62: 139-140.

Silva VNT, Oliveira FM, Conserva LM 2001. Phenolic derivatives and triterpenes from Acosmium bijugum. Biochem Syst Ecol 29: 1189-1192.

Simões CMO, Schenkel EP, Gosmann G, Mello JCP, Mentz LA, Petrovick PR 2000. Farmacognosia: da Planta ao Medicamento. Porto Alegre: UFRGS.

Sousa FCF, Melo CTV, Citó MCO, Félix FHC, Vasconcelos SMM, Fonteles MMF, Barbosa-Filho JM, Viana GSB 2008. Plantas medicinais e seus constituintes bioativos: Uma revisão da bioatividade e potenciais benefícios nos distúrbios da ansiedade em modelos animais. Rev Bras Farmacogn 18: 642-654.

Tolstikova TG, Sorokina IV, Tolstikova GA, Tolstikova AG, Flekhter OB 2006. Biological activity and pharmacological prospects of lupane terpenoids: I. Natural lupane derivatives Rus J Bioorg Chem 32: $37-49$.

Trevisan TC 2002. Estudo químico-farmacológico das cascas das raizes de Acosmium dasycarpum (Vog) Yakovlev. Cuiabá, 173 p. Dissertação de Mestrado - Programa de Pós-Graduação em Saúde Coletiva, Universidade Federal de Mato Grosso.

Veitch NC, Goodwin BL, Kite GC, Simmonds SJ 1997. Methoxylated quinolizidine alkaloids from Acosmium panamense. Phytochemistry 45: 847-850.

Vieira RA, Lapa AJ, Lima TCM 2000. Atividade do extrato etanólico de Acosmium subelegans (Mohlenbr) no sistema nervoso central (SNC) de camundongos. $X V I$ Simpósio de Plantas Medicinais do Brasil. Recife, Brasil. 